\title{
Phylogenetic analysis of Spirocerca lupi and Spirocerca vulpis reveal high genetic diversity and intra-individual variation
}

\author{
Alicia Rojas ${ }^{1}$, Eran Dvir ${ }^{2}$, Róbert Farkas ${ }^{3}$, Kalyan Sarma $^{4}$, Sonjoy Borthakur ${ }^{5}$, Abdul Jabbar $^{6}$, Alex Markovics ${ }^{7}$, \\ Domenico Otranto ${ }^{8}$ and Gad Baneth ${ }^{1 *}$ (D)
}

\begin{abstract}
Background: Spirocerca lupi is a parasitic nematode of canids that can lead to a severe and potentially fatal disease. Recently, a new species, Spirocerca vulpis, was described from red foxes in Europe, suggesting a high genetic diversity of the Spirocerca spp. infecting canids. The genetic variation and phylogenetic relationships of S. lupi collected from naturally-infected domestic dogs from Australia, Hungary, Israel, Italy, India and South Africa, and S. vulpis from red foxes from Bosnia and Herzegovina, Italy and Spain, was studied using mitochondrial and rDNA markers.

Results: A high intra-individual variation was found in the first internal transcribed spacer (ITS1) locus in all Spirocerca spp., ranging between $0.37-2.84 \%$, with up to six haplotypes per specimen. In addition, a combination of phylogenetic and haplotype analyses revealed a large variability between $S$. lupi specimens collected from different geographical locations using the ITS1 (0.37-9.33\%) and the cytochrome c oxidase subunit 1 (cox1) gene (1.42-6. 74\%). This genetic diversity led to the identification of two S. lupi genotypes circulating among dogs (PTP support $>0.829$ ), including genotype 1 found in S. lupi from Australia, India, Israel and South Africa, and genotype 2 represented by specimens from Hungary and Italy. These genotypes presented pairwise nucleotide distances of 0 . $14 \%, 8.06 \%$ and $6.48 \pm 0.28 \%$ in the small rDNA subunit (18S), ITS1 and cox1 loci, respectively. Additionally, Nei's genetic distance in the ITS1 showed a further subdivision of genotype 1 worms into 1A (Israel and South Africa) and 1B (Australia and India). A morphological analysis of the anterior and posterior extremities of genotype 1 and genotype 2 worms using scanning electron microscopy did not show any differences between the specimens, contrary to the morphological differences between S. lupi and S. vulpis.
\end{abstract}

Conclusions: These findings demonstrate the high genetic variability among Spirocerca spp. from different geographical locations, thereby expanding our understanding of the epidemiology, evolution and phylogenetic variability within the genus.

Keywords: Spirocerca lupi, Spirocerca vulpis, ITS1, cox1, 18S, Genetic variation

\section{Background}

Spirocerca lupi is a parasitic nematode that affects canids in tropical and subtropical regions around the world [1]. Its life-cycle includes coprophagous beetles and canids, as intermediate and definitive hosts, respectively. Among canids, dogs (Canis lupus familiaris) are

\footnotetext{
* Correspondence: gad.baneth@mail.huji.ac.il

${ }^{1}$ Koret School of Veterinary Medicine, The Hebrew University of Jerusalem,

Rehovot, Israel

Full list of author information is available at the end of the article
}

considered the main definitive hosts, while other wild canid species have been reported as potential alternative hosts for this parasite [2-4]. In dogs, S. lupi infection leads to spirocercosis, a life-threatening disease with clinical signs that range from regurgitation and vomiting of food, fatal hemothorax due to rupture of aortic aneurisms, neurological signs of spinal pain and paralysis due to spinal migration of the worm, and malignant transformation of oesophageal nodules with metastasis to other organs [1]. Recently, a new species of the genus

(c) The Author(s). 2018 Open Access This article is distributed under the terms of the Creative Commons Attribution 4.0 International License (http://creativecommons.org/licenses/by/4.0/), which permits unrestricted use, distribution, and 
Spirocerca was described from red foxes (i.e. Spirocerca vulpis), which is morphologically as well as genetically different from S. lupi and produces stomach nodules in these hosts [5]. The presence of this additional species in canids previously thought to be $S$. lupi highlights the need to further study the genetic composition and variability of this species.

Genetic diversity has been widely studied in other helminth species, such as Fasciola hepatica, Sabatieria spp. and Gongylonema pulchrum [6-8], leading to important implications about the evolutionary history and epidemiology dynamics of helminth infections. In the case of S. lupi, contrasting results have been obtained regarding the genetic composition in specimens from different parts of the world. For example, Traversa et al. [9] found low genetic variability in specimens of $S$. lupi obtained from Africa, Asia and Europe. In contrast, a study conducted in a limited area in South Africa found a high intra-host genetic variability and low variation within the analyzed area [10]. Similarly, a high genetic diversity was recently reported in S. lupi obtained from dogs and black backed jackals in South Africa [2] and from the Andean fox in Peru [11]. Furthermore, the analysis of three S. lupi populations from South Africa found a low population structure between groups and lack of panmixia [12]. The latter studies led to the suggestion of S. lupi as a species complex or the presence of cryptic and/or different Spirocerca spp. circulating among canid populations around the world [13]. In this regard, geographically isolated canid populations may lead to the genetic differentiation and divergence of parasite species through time [14]. Therefore, the genetic comparison of parasites obtained from different geographical locations or host species can assist in the delimitation of novel or cryptic species [15]. Thus, a deeper exploration of the genetic diversification of S. lupi and S. vulpis collected from different parts of the world is required to decipher the phylogenetic relationships between these specimens and determine the degree of genetic variability.

Mitochondrial and nuclear ribosomal DNA (rDNA) markers have extensively been used for phylogenetic and diversity studies in several parasite species $[15,16]$. The rRNA genes are usually encoded in the genome in multiple tandem copies, which include conserved coding regions (i.e. $18 S, 5.8 S$ and $28 S$ genes) separated by highly variable non-coding spacers known as the first and second internal transcribed spacers (ITS1 and ITS2, respectively) or intergenic spacers [17]. In helminths, the characterization of the ITS1 and ITS2 loci has shown a high variability between species within the same genera [18], between individuals of the same species [19] or even within a single adult worm $[20,21]$. This characteristic makes the ITS sequences suitable targets for phylogenetic comparisons among nematodes of the same species from different geographical and host origins [22]. However, the ITS1 of S. lupi has not been characterized to date and no analyses comparing specimens from different countries have been assessed.

The purpose of the present study was to analyze the phylogeny, genetic variability and diversity of $S$. lupi from dogs collected from six countries in four different continents, and S. vulpis obtained from red foxes collected from three European countries, using three different genetic markers (i.e. ITS1, $18 S$ and cox1). This study reveals the existence of two major S. lupi genotypes distributed according to geographical locations and shows the high genetic diversity present in the genus. Overall, these results highlight the utility of these markers for further genetic population and evolutionary studies.

\section{Methods}

\section{Specimen collection and DNA extraction}

A total of $31 \mathrm{~S}$. lupi and S. vulpis specimens were obtained from dogs and red foxes from eight countries (Table 1). All the specimens from dogs were obtained from oesophageal nodules after post-mortem dissection of the animals. The worms from foxes were collected from nodules localized in the stomach wall from authorized captures, including wildlife protection centers or from road-killed animals after car accidents. Details on the year of collection, the number of worms processed from each collection site and the code assigned for each worm specimen are included in Table 1.

DNA was extracted from approximately $1 \mathrm{~cm}$ of the worms' body using the DNeasy Blood \& Tissue kit (Qiagen, Hilden, Germany) following the manufacturer's instructions.

\section{Amplification of the 18S, ITS1 and cox1 loci}

The target loci, PCR primers and their concentrations, and the conditions for PCR amplification are specified in Table 2 and in Additional file 1: Figure S1. All reactions for amplifying the $18 S$, ITS1 and cox 1 loci were run in a final volume of $25 \mu \mathrm{l}$ containing primers at different concentrations, $150 \mathrm{ng}$ of DNA template, and $20 \mu \mathrm{l}$ of ultra-pure water (UPW) in ready-to-use PCR tubes containing a dehydrated master mix (Syntezza Bioscience Ltd., Jerusalem, Israel). In addition, all PCRs in this study included positive (S. lupi-DNA), negative (Leishmania infantum-DNA) and non-template (UPW) controls. All amplicons obtained were examined on 2\% agarose gels stained with ethidium bromide. Accordingly, a $1611 \mathrm{bp}$ fragment of the $18 \mathrm{~S}$ gene was obtained by joining the outcomes of two separate conventional PCRs using two different sets of primers (Table 2, Additional file 1: Figure S1) [23]. 
Table 1 Summary of data on Spirocerca spp. adult worms included in the study

\begin{tabular}{|c|c|c|c|c|c|}
\hline Species & Country of origin & Year of collection & Host & No. of specimens & Code \\
\hline \multirow[t]{10}{*}{ S. lupi } & \multirow[t]{4}{*}{ Israel } & 1993 & Dog & 3 & $\mathrm{~A}, \mathrm{G}, \mathrm{H}^{\mathrm{a}}$ \\
\hline & & 2004 & Dog & 1 & B \\
\hline & & 2013 & Dog & 1 & C \\
\hline & & 2015 & Dog & 3 & $D, E, F^{a}$ \\
\hline & \multirow[t]{2}{*}{ South Africa } & 2009 & Dog & 3 & $\mathrm{I}, \mathrm{J}, \mathrm{K}$ \\
\hline & & 2010 & Dog & 1 & L \\
\hline & India & 2016 & Dog & 5 & $M, N, O, P, Q$ \\
\hline & Italy & 2013 & Dog & 1 & $\mathrm{R}$ \\
\hline & Australia & 1989 & Dog & 2 & $\mathrm{~V}, \mathrm{~W}^{\mathrm{a}}$ \\
\hline & Hungary & 2016 & Dog & 4 & $\mathrm{AA}, \mathrm{BA}, \mathrm{CA}, \mathrm{DA}^{\mathrm{a}}$ \\
\hline \multirow[t]{3}{*}{ S. vulpis } & Spain & 2015 & Red fox & 3 & $\mathrm{~S}, \mathrm{~T}, \mathrm{U}$ \\
\hline & Italy & 2015 & Red fox & 1 & $x$ \\
\hline & Bosnia and Herzegovina & 2016 & Red fox & 3 & $E A, F A, G A$ \\
\hline
\end{tabular}

${ }^{a}$ These worms are siblings, i.e. were recovered from the same dog

Table 2 Summary of the gene targets, primers, PCR conditions employed for the amplification and genetic analysis of Spirocerca spp.; and the outgroups used for the phylogenetic analyses

\begin{tabular}{|c|c|c|c|c|}
\hline $\begin{array}{l}\text { Gene } \\
\text { target }\end{array}$ & $\begin{array}{l}\text { Amplicon } \\
\text { length } \\
\text { (bp) }\end{array}$ & $\begin{array}{l}\text { Primers (final concentration used in the } \\
\text { PCR) [reference] }\end{array}$ & PCR conditions & Outgroups employed in phylogenetic analysis \\
\hline \multirow[t]{2}{*}{185} & $\sim 750$ & $\begin{array}{l}\text { Nem18S-F (5'-CGC GAA TRG CTC ATT } \\
\text { ACA ACA GC-3') and Nem18S-R (5'-GGG } \\
\text { CGG TAT CTG ATC GCC-3') (400 nM) [23] }\end{array}$ & $\begin{array}{l}95^{\circ} \mathrm{C} \text { for } 5 \text { min; } 35 \text { cycles of } 95^{\circ} \\
\mathrm{C} \text { for } 1 \text { min, } 56^{\circ} \mathrm{C} \text { for } 1 \text { min and } \\
72{ }^{\circ} \mathrm{C} \text { for } 1 \text { min; } 72^{\circ} \mathrm{C} \text { for } 5 \text { min }\end{array}$ & \multirow{2}{*}{$\begin{array}{l}\text { Spirocerca lupi (AY751497.1 and Q674750.1), } \\
\text { Spirocerca sp. (AY751498.1), Cylicospirura petrowi } \\
\text { (KM434335.1), Gongylonema pulchrum } \\
\text { (AB495401.2), Gongylonema nepalensis } \\
\text { (AB646109.1), Oxyspirura petrowi (LC316613.1), } \\
\text { Dirofilaria immitis (AF182647.1), Dirofilaria repens } \\
\text { (AB973229.1), Loa loa (XR002251421.1), Wuchereria } \\
\text { bancrofti (AY843436.1), Litosomoides sigmodontis } \\
\text { (AF227233.1), Thelazia lacrymalis (DQ503458.1), } \\
\text { Thelazia callipaeda (AB538282.1) }\end{array}$} \\
\hline & $\sim 870$ & $\begin{array}{l}\text { Nem18S-F2 (5'-CGA AAG TCA GAG GTT } \\
\text { CGA AGG-3') and Nem18S-R2 (5'-AAC CTT } \\
\text { GTT ACG ACT TाT GCC C-3') (400 nM) [5] }\end{array}$ & $\begin{array}{l}95^{\circ} \mathrm{C} \text { for } 5 \text { min; } 35 \text { cycles of } 95^{\circ} \\
\mathrm{C} \text { for } 1 \text { min, } 56^{\circ} \mathrm{C} \text { for } 1 \text { min and } \\
72{ }^{\circ} \mathrm{C} \text { for } 1 \text { min; } 72^{\circ} \mathrm{C} \text { for } 5 \text { min }\end{array}$ & \\
\hline \multirow[t]{3}{*}{ ITS1 } & 610 & $\begin{array}{l}\text { rDNA2 (5'-TTG ATT ACG TCC CTG CCC } \\
\text { TIT-3') [24] and rDNA1.58S (5'-GCC ACC } \\
\text { TAG TGA GCC GAG CA-3') (375 nM) [25] }\end{array}$ & $\begin{array}{l}94^{\circ} \mathrm{C} \text { for } 5 \text { min; } 35 \text { cycles of } 94^{\circ} \\
\mathrm{C} \text { for } 1 \text { min, } 60^{\circ} \mathrm{C} \text { for } 1 \text { min and } \\
72{ }^{\circ} \mathrm{C} \text { for } 2 \text { min; } 72{ }^{\circ} \mathrm{C} \text { for } 5 \text { min }\end{array}$ & \multirow[t]{3}{*}{ C. petrowi (KM434335.1) } \\
\hline & $\sim 100$ & $\begin{array}{l}\text { ITS inner-F (5'-GCT ATC TTG TAA AAA } \\
\text { CGG TG-3') and M13-R (5'-CAG GAA ACA } \\
\text { GCT ATG AC-3') (200 nM) }\end{array}$ & $\begin{array}{l}94^{\circ} \mathrm{C} \text { for } 5 \text { min; } 35 \text { cycles of } 94^{\circ} \\
\mathrm{C} \text { for } 1 \text { min, } 60^{\circ} \mathrm{C} \text { for } 1 \text { min and } \\
72{ }^{\circ} \mathrm{C} \text { for } 2 \text { min; } 72{ }^{\circ} \mathrm{C} \text { for } 5 \text { min }\end{array}$ & \\
\hline & 450 & $\begin{array}{l}\text { C18 (5'-GTT TCC GTA GGT GAA CCT GC- } \\
\left.3^{\prime}\right) \text { and } 5818\left(5^{\prime}-\text { ACG ARC CGA GTG ATC }\right. \\
\text { CAC-3') (400 nM) [26] }\end{array}$ & $\begin{array}{l}94^{\circ} \mathrm{C} \text { for } 4 \text { min; } 40 \text { cycles of } 94^{\circ} \\
\mathrm{C} \text { for } 45 \mathrm{~s}, 59^{\circ} \mathrm{C} \text { for } 30 \mathrm{~s} \text { and } 72 \\
{ }^{\circ} \mathrm{C} \text { for } 2 \mathrm{~min} ; 72^{\circ} \mathrm{C} \text { for } 5 \text { min }\end{array}$ & \\
\hline \multirow[t]{3}{*}{$\cos 1$} & $\begin{array}{l}650 \\
\text { (Fragment } \\
\text { A) }\end{array}$ & $\begin{array}{l}\text { NTF (5'-TGA TTG GTG GTT TTG GTA A-3') } \\
\text { and NTR (5'-ATA AGT ACG AGT ATC AAT } \\
\text { ATC-3') (200 nM) [28] }\end{array}$ & $\begin{array}{l}95^{\circ} \mathrm{C} \text { for } 2 \mathrm{~min} ; 49 \text { cycles of } 95^{\circ} \\
\mathrm{C} \text { for } 1 \mathrm{~min}, 54^{\circ} \mathrm{C} \text { for } 1 \mathrm{~min}, 72^{\circ} \\
\mathrm{C} \text { for } 1 \mathrm{~min} ; 72^{\circ} \mathrm{C} \text { for } 7 \mathrm{~min}\end{array}$ & \multirow[t]{2}{*}{$\begin{array}{l}\text { S. lupi from China (KC305876.1) [27], Spirocerca sp. } \\
\text { from Denmark (KJ605484) [13], C. petrowi } \\
\text { (KF719952.1), Cylicospirura felineus (GQ342967.1) } \\
\text { [61], Cylicospirura subaequalis (GQ342968.1) [60] }\end{array}$} \\
\hline & $\sim 300$ & $\begin{array}{l}\text { NTInt (5'-GGC TAG ACA ACT CTA AAC G- } \\
\left.3^{\prime}\right)^{\mathrm{a}} \text { and NTF (5'-TGA TTG GTG GTT TTG } \\
\text { GTA A-3') (200 nM) [28] }\end{array}$ & $\begin{array}{l}95^{\circ} \mathrm{C} \text { for } 2 \text { min; } 49 \text { cycles of } 95^{\circ} \\
\mathrm{C} \text { for } 1 \text { min, } 54^{\circ} \mathrm{C} \text { for } 1 \text { min, } 72^{\circ} \\
\mathrm{C} \text { for } 1 \text { min; } 72^{\circ} \mathrm{C} \text { for } 7 \text { min }\end{array}$ & \\
\hline & $\begin{array}{l}394 \\
\text { (Fragment } \\
\text { B) }\end{array}$ & $\begin{array}{l}\text { JB3 (5'-TTा TाT GGG CAT CCT GAG GTT } \\
\text { TAT-3') and JB4.5 (5'-TAA AGA AAG AAC } \\
\text { ATA ATG AAA ATG-3') (400 nM) [30] }\end{array}$ & $\begin{array}{l}95^{\circ} \mathrm{C} \text { for } 5 \text { min; } 30 \text { cycles of } 95^{\circ} \\
\mathrm{C} \text { for } 1 \text { min, } 54^{\circ} \mathrm{C} \text { for } 1 \text { min and } \\
72{ }^{\circ} \mathrm{C} \text { for } 1 \text { min; } 72{ }^{\circ} \mathrm{C} \text { for } 5 \text { min }\end{array}$ & $\begin{array}{l}\text { S. lupi from China (KC305876.1) [27], S. lupi } \\
\text { obtained from domestic dogs and black-backed } \\
\text { jackals from South Africa (KY495493.1-KY495505.1) } \\
\text { [2] and from the Andean fox from Peru } \\
\text { (KY634868.1-KY634870.1) [11] }\end{array}$ \\
\hline
\end{tabular}


The complete ITS1 locus of the Spirocerca-adults was amplified by conventional PCR using primers rDNA2 [24] and rDNA1.58S [25] (Table 2, Additional file 1: Figure S1). In case of negative results in this reaction, an additional PCR was performed using the C18 and 5818 primers [26] (Table 2). Preliminary sequencing of the ITS1 amplicons, suggested the presence of multiple different ITS1 copies within each specimen (i.e. unresolved chromatograms). Thus, all ITS1 PCR amplicons were cloned into plasmids in competent bacterial cells for further individual amplification and sequencing. Accordingly, the amplicons were excised and purified from agarose gels using the NucleoSpin ${ }^{\circledR}$ Gel and PCR Clean-up kit (Macherey-Nagel GmbH \& Co., Düren, Germany). Subsequently they were cloned into pCR 2-TOPO vectors using the Invitrogen TOPO TA cloning kit (Life Technologies, Thermo Fisher Scientific Inc, Waltham, USA) with the white-blue colony screening protocol, according to the manufacturer's instructions. To allow determination of different ITS1 copies per individual, plasmids were extracted from 12 different colonies per nematode using the FB Plasmid Miniprep kit (FairBiotech Corp., Taoyuan, China). Subsequently, the cloned fragments were amplified by conventional PCR using the universal primers M13-F and M13-R provided with the kit. In cases where unresolved sequences were obtained, the ITS1 clones were further tested in a conventional PCR with the designed primer ITS inner-F and M13-R (Table 2). Finally, the amplicons were sequenced as described below.

The cox 1 gene was amplified using different primer sets to allow the comparison of the study specimens with sequences from different organisms available on GenBank, as follows. First, a 650 bp fragment (positions 317 to 967 bp of the cox 1 gene; [27], hereinafter referred to as cox 1 fragment A) was amplified using the NTF and NTR primers [28] (Table 2, Additional file 1: Figure S1), which allowed comparison with some Spirocerca spp. and other Cylicospirura spp. sequences. Larger than expected amplicons ( 1000 bp) were obtained from several specimens, which amplified an additional 300 bp-upstream region from the cox1 fragment A (demonstrated by sequencing analysis; Additional file 1: Figure S1, Additional file 2: Figure S2). To confirm the latter, a reverse primer, identified as NTInt was designed using Primer-BLAST [29] and run together with the NTF primer in an additional PCR (Table 2). Secondly, a 394 bp fragment from positions 807 to 1201 bp of the cox 1 gene (hereinafter referred to as cox 1 fragment B) was amplified using primers JB3 and JB4.5 [30] (Table 2, Additional file 1: Figure S1) to compare the study's sequences with other $S$. lupi sequences available on GenBank.

\section{DNA sequencing of amplicons}

All DNA amplicons were purified using the Exo-SAP mix (New England Bio-Labs Inc., Ipswich, USA) and sequenced using primers in both directions in the Big-Dye Terminator cycle sequencing chemistry from Applied Biosystems ABI3700 DNA Analyzer and the ABI's Data Collection and Sequence Analysis software (Applied Biosystems, Thermo Fisher Scientific Inc., Waltham, USA).

\section{Sequence analyses}

All sequences were manually inspected, cleaned of nucleotide ambiguities and trimmed from the primer sequences using the MEGA 7.0 software [31]. Each indel and/or single nucleotide substitution present in the sequences were double-checked by manual verification in the sequence chromatogram and re-sequenced in case of ambiguity. The $18 S$, ITS1 and $\operatorname{cox} 1$ sequences obtained in this study were aligned together with sequences for other Spirocerca spp. and nematode outgroups (specified in Table 2), in accordance with their GenBank availability, using the ClustalW algorithm with conventional gap opening and extension penalty of 15 and 6.66.

The boundaries of the ITS1 of Spirocerca spp. (i.e. the $18 S$ downstream and the $5.8 S$ upstream regions) were determined using the outgroup sequences (Table 2) and Thelazia spp. sequences [32]. The $\mathrm{G}+\mathrm{C}$ content was calculated using the ENDMEMO webtool (http:// www.endmemo.com/bio/gc.php) and microsatellites were searched for using the Microsatellite repeats finder webtool (http://insilico.ehu.es/mini_tools/microsatellites/ ) with 2 to 6 bp sequence length and a minimum of 4 repeats. An unpaired t-test (Excel 365 software, Microsoft, Redmont, USA) was employed to determine differences in the length and $\mathrm{G}+\mathrm{C}$ content of ITS1 sequences obtained from different specimens of Spirocerca spp. The Bonferroni correction was applied in multiple comparisons and significance was determined when $P<0.003$.

Both overlapping cox 1 fragments obtained in our specimens were assembled to generate an 888 bp contiguous sequence, which was compared to D. immitis (GenBank: AJ537512.1) and used for further analyses.

\section{Genetic diversity, differentiation and distance analyses}

The number of haplotypes $(h)$, haplotype diversity $(\mathrm{Hd})$, segregating sites $(\mathrm{S})$, and nucleotide diversity $(\pi)$ were calculated for the ITS 1 and the 888 bp-assembled cox 1 fragment using DnaSP 5.10.01 [33] with 10,000 permutations. The gaps generated in the ITS1 alignment were not included in this analysis. Additionally, the ITS1 and cox1 genetic distances between sampled individuals was calculated separately according to Nei [34]. A principal coordinate analysis (PCoA) was drawn using the GenAIEx 6.5 software [35]. Furthermore, the pairwise 
nucleotide p-distances of the ITS1, $\operatorname{cox} 1$ (A, B and combined fragments) and $18 S$ sequences were calculated using the MEGA 7.0 software [31]. Complete deletion was assumed in sites with gaps. In the ITS1 analysis, the generated sequences from each specimen were compared between each other with the median and third interquartile ranges (3rd IQR). All ITS1, $18 S$ and cox1 sequence novel genetic variants detected in this study were deposited in the GenBank database.

\section{Phylogenetic and species delimitation analyses}

The phylogenetic relationships of the ITS1 and cox1 (with the 888 bp-assembled fragment) haplotypes were analyzed by building a haplotype network for each locus separately. A Templeton-Crandall-Sing (TCS) haplotype network was drawn with the method of statistical parsimony [36] and a 95\% connection limit ignoring sites with gaps using the PopART software (available at http://popart.otago.ac.nz).

The ITS1, $18 S$ and 888 bp-cox1 alignments were analyzed using Bayesian inference (BI) and Maximum Likelihood (ML) phylogenetic algorithms. First, the best nucleotide substitution model for the BI tree was chosen according to the Akaike information criterion (AIC) as determined by JModelTest 2.1.6 [37] implemented on the XSEDE server of the Cipres Science Gateway [38]. Ultrametric trees were generated with the Bayesian Evolutionary Analysis by Sampling Trees (BEAST) package 1.8.4. The program Bayesian Evolutionary Analysis Utility (BEAUti) was used to generate the input XML file with a Markov Chain Monte Carlo (MCMC) analysis run for $10^{7}$ generations, a sampling frequency of every $10^{3}$ generated trees, $10^{6}$ states of 'burn-in' length and a relaxed lognormal clock. The nucleotide substitution models Hasegawa-Kishino-Yano with gamma distribution $(\mathrm{HKY}+\mathrm{G})(\gamma=4)$, the three parameters with gamma distribution $(\mathrm{TPM}+\mathrm{G})(\gamma=4)$ and the transitional model with gamma distribution $(\mathrm{TIM}+\mathrm{G})(\gamma=4)$ were implemented for the ITS1, $18 S$ and cox 1 databases, respectively. The convergence of the chains was verified by the effective sample sizes (ESS) with values larger than 300 in all priors using Tracer 1.6.0. The generated trees for all loci were summarized with TreeAnotator 1.8 .4 and the consensus trees were visualized using FigTree 1.4.3. Secondly, the ML tree was generated using the PhyML online execution program (http://www.atgc-montpellier.fr/phyml/) [39] with bootstrap replicates set to $10^{3}$. In addition, the nucleotide substitution model was selected according to the AIC using the Smart Model Selection (SMS) in the same website [40]. The chosen models were HKY $+\mathrm{G}(\gamma=4)$ for the ITS1 and $\operatorname{cox} 1$ databases, and the general time reversible with gamma distribution and invariant sites $(\mathrm{GTR}+\mathrm{G}+\mathrm{I})(\gamma=4)$ for the $18 S$ alignment. The outgroup sequences used for each tree are specified in Table 2.

Finally, the Poisson Tree Processes (PTP) [41] for species delimitation were used to identify the most likely number of species present in the samples using the ITS1 locus since this database included all sampled specimens. In the PTP model, speciation events are hypothesized according to the observed number of substitutions between sequences, using either a maximum likelihood (mPTP) or Bayesian solution (bPTP). The input Newick file was generated in FigTree from the BI phylogenetic tree used in the analysis described above. The algorithm was implemented on the website http://species.h-its.org/ptp/ with $10^{5}$ MCMC generations, 'burn-in' length of $10^{4}$ and a thinning of $10^{2}$. Convergence of the MCMC chains were visually inspected to confirm the equilibrium distribution.

\section{Scanning electron microscopy (SEM) analysis}

To evaluate potential morphological differences in a novel identified S. lupi genotype, SEM analysis was performed to visualize the anterior and posterior extremities of four worms collected from dogs from Hungary. The worms were washed, fixed and dehydrated in an ethanol series as previously described [5]. Then, the worms were dried with a critical point dryer (Quorum K850, Quorum Technologies Ltd., Lewes, UK) with $\mathrm{CO}_{2}$, mounted on aluminum stubs, coated with iridium for $20 \mathrm{~s}$ (Quorum Spatter coater Q150T ES, Quorum Technologies Ltd.) and observed in a scanning electron microscope (JSM-IT100, JEOL USA Inc., Peabody, USA) in a $10 \mathrm{~mm}$ stage height operated at $3 \mathrm{kV}$. The obtained SEM images were compared to those from $S$. lupi and $S$. vulpis recently reported [5].

\section{Results}

\section{Analysis of the $18 \mathrm{~S}$ gene}

The $18 S$ gene (1611 bp) was successfully amplified from $87 \%(27 / 31)$ of the nematodes. The comparison of this locus showed nucleotide distances below $1 \%$ between S. lupi and S. vulpis (Additional file 3: Table S1). Nevertheless, three groups of genotypes (i.e. each containing identical sequences) were identified among the specimens, as follows: (i) S. lupi genotype $1(n=17)$ from dogs from Israel, South Africa and India; (ii) $S$. lupi genotype $2(n=4)$ from dogs only from Hungary; and (iii) S. vulpis $(n=6)$ from red foxes from Spain and Bosnia and Herzegovina specimens. Five polymorphic nucleotide sites were observed within the S. lupi genotypes 1 and 2, that included two indels, two nucleotide transitions ( $\mathrm{C}$ to $\mathrm{T}$ and from $\mathrm{A}$ to $\mathrm{G}$ ) and a transversion ( $\mathrm{G}$ to $\mathrm{C}$ ). The nucleotide distance between S. lupi genotype 1 and genotype 2 was $0.187 \%$, while both $S$. lupi genotypes differed from S. vulpis by $0.062 \%$. Spirocerca 
lupi genotypes 1 and 2 were $99 \%$ identical to a reference sequence of S. lupi (GenBank: AY751497.1). In addition, the distance between $S$. lupi genotype 1, S. lupi genotype 2 and S. vulpis to a Spirocerca sp. collected from the Island fox (Urocyon littoralis) from San Miguel Island in the USA ranged between 1.721-1.849\%. Nucleotide pairwise distances of Spirocerca spp. analyzed herein with other members of the Spiruridae family ranged between 3.783-11.289\% (Additional file 3: Table S1). An $18 S$ sequence from $S$. lupi genotype 2 was deposited in the GenBank database under the accession number MH628159.

The ML and BI phylogenetic trees resulted in identical topologies (Fig. 1). Notably, both trees clustered all Spirocerca spp. together, with high bootstrap values $(71 \%)$ and posterior probabilities (0.892). Nevertheless, Spirocerca spp. associated internal nodes were less supported, with the exception of the cluster of S. vulpis and S. lupi genotype 2 sequences (Fig. 1). Moreover, the separation of all Spirocerca spp. from other members of the family Spiruridae such as C. petrowi, Gongylonema spp. and Oxyspirura petrowi was evident, with high support values.

\section{Analysis of the ITS1 loci}

Intra-individual variability in the ITS1 loci was detected in both Spirocerca spp. A total of 264 ITS1 sequences were obtained and analyzed from the 31 worms, i.e. 210 for S. lupi and 54 for S. vulpis (Table 3). Each individual nematode contained two or more different copies (up to 6 different copies per individual) of the ITS1 locus (hereinafter referred to as "haplotypes"), with sequence variations that ranged between $0.37-2.85 \%$ (Table 3). The worms with the smallest and largest median intra-individual variation were the $S$. lupi specimens N, $\mathrm{O}$ and $\mathrm{P}$ from India (with all sequences, except one, identical to each other) and the S. lupi specimen CA from Bosnia and Herzegovina (median $=1.47$, 3rd interquartile range (IQR): 1.45-1.49), respectively. The intra-individual variation from $S$. vulpis specimens ranged between $0.37-1.80 \%$ (Table 3). The S. lupi sequences from Israel, South Africa, India and Australia were $96 \%$ identical to the $S$. lupi reference ITS1 sequence (GenBank: MF425539), described earlier [42]. Additionally, S. lupi sequences of worms collected from Hungarian and Italian dogs and S. vulpis were $72 \%$ and $64 \%$ identical to this same reference sequence.

The $\mathrm{G}+\mathrm{C}$ content and length of S. lupi ITS1 sequences differed between the different geographical locations (Table 4). First, pairwise comparisons showed significant differences in the length of the $S$. lupi sequences between most of the paired geographical locations (all

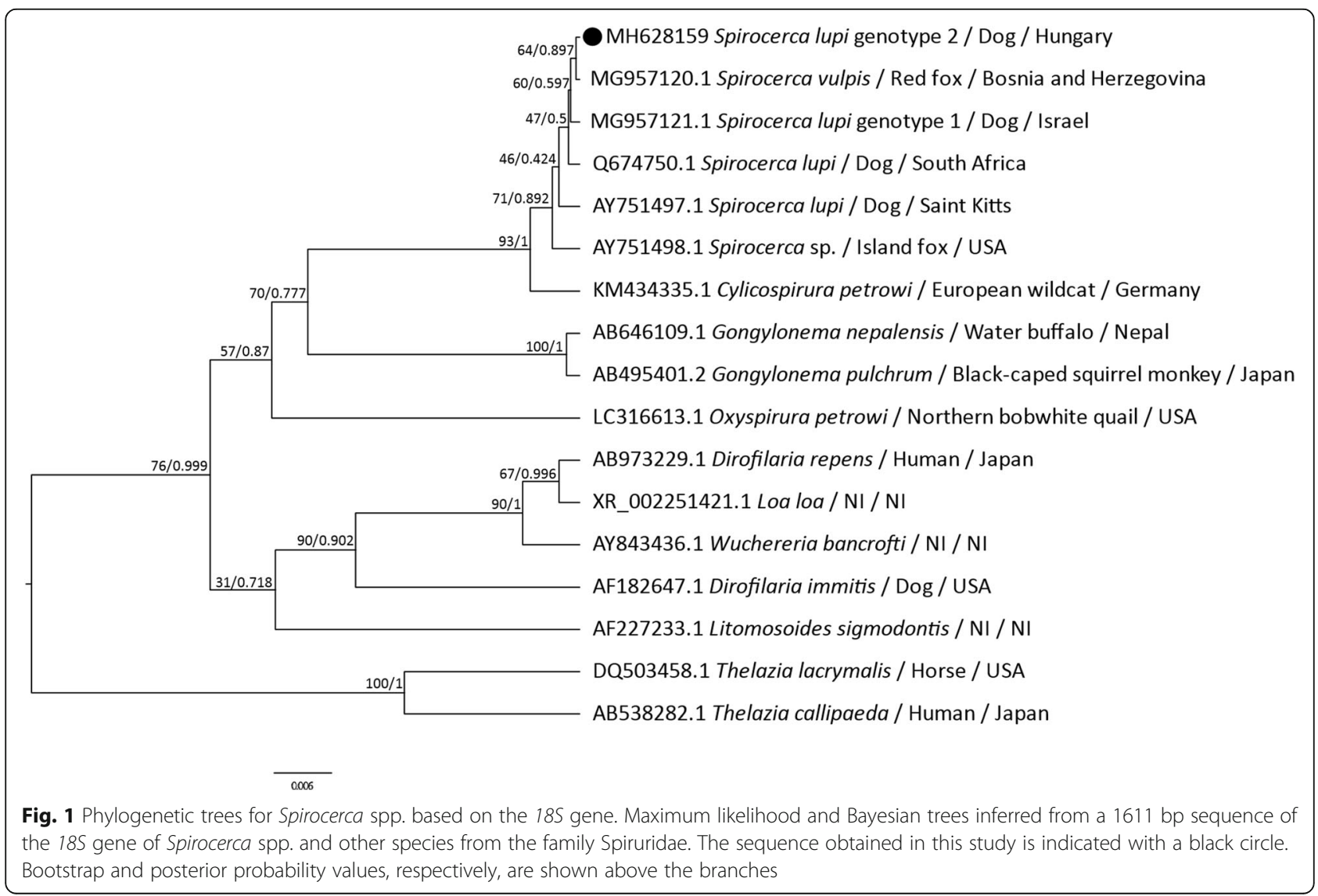


Table 3 Intra-individual pairwise nucleotide distances (\%) of the ITS1 sequences of Spirocerca spp.

\begin{tabular}{|c|c|c|c|c|}
\hline Specimen & No. of sequences analyzed & Median (third interquartile range) & Minimal variation & Maximal variation \\
\hline \multicolumn{5}{|l|}{ S. lupi } \\
\hline A & 10 & $0.37(0.36-0.38)$ & 0.37 & 0.71 \\
\hline B & 10 & $0.37(0.36-0.38)$ & 0.37 & 0.71 \\
\hline C & 9 & $0.73(0.72-0.74)$ & 0.37 & 1.42 \\
\hline D & 11 & $0.73(0.71-0.75)$ & 0.37 & 2.85 \\
\hline$E^{a}$ & 8 & $0.37(0.30-0.44)$ & 0.73 & 1.78 \\
\hline $\mathrm{F}$ & 7 & $0.37(0.30-0.44)$ & 0.37 & 0.71 \\
\hline G & 10 & $0.73(0.72-0.74)$ & 0.37 & 1.42 \\
\hline $\mathrm{H}$ & 8 & $0.73(0.72-0.74)$ & 0.37 & 1.42 \\
\hline । & 10 & $0.74(0.73-0.75)$ & 0.37 & 1.07 \\
\hline J & 8 & $0.73(0.723-0.80)$ & 0.37 & 1.07 \\
\hline K & 8 & $0.73(0.72-0.74)$ & 0.37 & 0.71 \\
\hline L & 7 & $0.73(0.71-0.75)$ & 0.37 & 2.17 \\
\hline M & 10 & $0.37(0.36-0.38)$ & 0.37 & 1.07 \\
\hline$N^{a}$ & 9 & 0 & - & 0.71 \\
\hline $\mathrm{O}^{\mathrm{a}}$ & 7 & 0 & - & 0.71 \\
\hline$P^{a}$ & 8 & 0 & - & 0.71 \\
\hline Q & 8 & $0.37(0.36-0.38)$ & 0.37 & 0.71 \\
\hline V & 11 & $0.73(0.72-0.74)$ & 0.37 & 1.78 \\
\hline W & 5 & $1.10(1.08-1.12)$ & 0.37 & 1.78 \\
\hline $\mathrm{R}$ & 12 & $0.55(0.54-0.56)$ & 0.37 & 1.43 \\
\hline AA & 10 & $0.74(0.73-0.75)$ & 0.37 & 1.79 \\
\hline BA & 10 & $1.10(1.08-1.12)$ & 0.37 & 2.14 \\
\hline CA & 8 & $1.47(1.45-1.49)$ & 0.37 & 1.43 \\
\hline DA & 6 & $1.10(1.08-1.12)$ & 0.37 & 2.14 \\
\hline \multicolumn{5}{|l|}{ S. vulpis } \\
\hline S & 10 & $0.37(0.36-0.38)$ & 0.37 & 1.80 \\
\hline$T^{\mathrm{a}}$ & 3 & $0.37(0.36-0.38)$ & 0.37 & 0.72 \\
\hline$U$ & 7 & $0.73(0.72-0.74)$ & 0.37 & 1.42 \\
\hline$x$ & 11 & $0.37(0.36-0.38)$ & 0.37 & 1.42 \\
\hline EA & 7 & $0.37(0.36-0.38)$ & 0.37 & 1.07 \\
\hline FA & 6 & $0.37(0.36-0.38)$ & 0.37 & 0.72 \\
\hline GA & 10 & $0.37(0.36-0.38)$ & 0.37 & 1.08 \\
\hline
\end{tabular}

${ }^{a}$ All sequences of the specimen, except for one, were identical to each other

$P<0.000001)$, except for South Africa and Australia, India and Australia, and, Hungary and Italy. In addition, the $\mathrm{G}+\mathrm{C}$ content varied significantly between the worms from Israel and the other geographical locations (all $P<$ 0.00001 ), and between India and the locations of Australia and Hungary $(P=0.00002$ and 0.0005 , respectively). In the case of $S$. vulpis, the $\mathrm{G}+\mathrm{C}$ content and sequence lengths did not show significant differences between the sampling locations (all $P>0.05$ ). Moreover, there were no differences when comparing the averages of these parameters between $S$. vulpis and $S$. lupi sequences $(P=0.031$; not significant after correction for multiple comparisons).

Five microsatellite motifs were found in the ITS1 sequences of both Spirocerca spp., namely $(\mathrm{AA})_{4},(\mathrm{AT})_{4}$, $(\mathrm{TA})_{5},(\mathrm{GT})_{4,6},(\mathrm{AC})_{4-11}$ and $(\mathrm{TA})_{4-6}$. Of these, microsatellite (AC) was present in $88.6 \%(186 / 210)$ of the $S$. lupi ITS1 sequences and was found in four copies in the specimens from Israel, South Africa, India and Australia; and six to eleven copies in the worms from Hungary and Italy. In addition, microsatellite $(\mathrm{TA})_{5}$ was observed in $100 \%(100 / 100)$ of the sequences of S. vulpis and S. lupi 
Table 4 General characteristics of the ITS1 sequences of Spirocerca spp. according to the geographical location

\begin{tabular}{|c|c|c|c|c|c|c|}
\hline Geographical location & $\begin{array}{l}\mathrm{G}+\mathrm{C} \text { content }(\%) \\
\text { Mean } \pm \mathrm{SD}\end{array}$ & $\begin{array}{l}\text { Length (bp) } \\
\text { Mean } \pm \text { SD }\end{array}$ & $S$ & $h$ & $\mathrm{Hd} \pm \mathrm{SD}$ & $\pi \pm S D$ \\
\hline \multicolumn{7}{|l|}{ S. lupi } \\
\hline Israel $(n=73)^{\mathrm{a}, \mathrm{b}}$ & $30.24 \pm 0.82$ & $398 \pm 6$ & 27 & 22 & $0.874 \pm 0.026$ & $0.0074 \pm 0.0006$ \\
\hline South Africa ${ }^{a, b}(n=34)$ & $31.56 \pm 0.96$ & $387 \pm 12$ & 9 & 8 & $0.886 \pm 0.014$ & $0.0064 \pm 0.0008$ \\
\hline India $(n=41)^{\mathrm{b}}$ & $31.12 \pm 1.02$ & $380 \pm 5$ & 15 & 7 & $0.263 \pm 0.049$ & $0.0026 \pm 0.0012$ \\
\hline Australia $(n=16)^{\mathrm{b}}$ & $31.95 \pm 0.35$ & $379 \pm 2$ & 9 & 9 & $0.825 \pm 0.036$ & $0.0051 \pm 0.0010$ \\
\hline Italy $(n=12)^{c}$ & $31.43 \pm 0.70)$ & $371 \pm 2$ & 5 & 6 & $0.758 \pm 0.079$ & $0.0050 \pm 0.0014$ \\
\hline Hungary $(n=34)^{c}$ & $31.79 \pm 0.51$ & $357 \pm 8$ & 11 & 10 & $0.722 \pm 0.016$ & $0.0055 \pm 0.0027$ \\
\hline Total $(n=210)$ & $31.11 \pm 1.05$ & $383 \pm 17$ & - & - & - & - \\
\hline \multicolumn{7}{|l|}{ S. vulpis } \\
\hline Spain $(n=20)^{d}$ & $31.33 \pm 0.34$ & $390 \pm 3$ & 5 & 6 & $0.445 \pm 0.024$ & $0.0022 \pm 0.0020$ \\
\hline Italy $(n=11)^{\mathrm{d}}$ & $31.21 \pm 0.47$ & $391 \pm 4$ & 3 & 3 & $0.345 \pm 0.107$ & $0.0024 \pm 0.0013$ \\
\hline Bosnia and Herzegovina $(n=23)^{d}$ & $31.45 \pm 0.30$ & $390 \pm 5$ & 4 & 4 & $0.249 \pm 0.020$ & $0.0015 \pm 0.0007$ \\
\hline Total $(n=54)$ & $31.36 \pm 0.36$ & $392 \pm 4$ & - & - & - & - \\
\hline
\end{tabular}

${ }^{a}$ Contains one haplotype shared with Israeli and South African sequences

${ }^{b}$ Contains one haplotype shared by Israeli, South African, Indian and Australian sequences

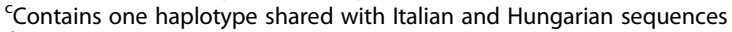

${ }^{d}$ Contains one haplotype shared with Spanish, Italian and Bosnian sequences

Abbreviations: $\mathrm{n}$, number of obtained sequences; $\mathrm{S}$, segregating sites; $h$, number of haplotypes; Hd, haplotype diversity; $\pi$, nucleotide diversity; bp, base pairs; SD, standard deviation

specimens from Hungary and Italy. Microsatellite $(\mathrm{GT})_{4,6}$ was found in $89 \%$ (48/54) of the S. vulpis specimens.

Haplotype and nucleotide diversity parameters of the ITS1, Hd and $\pi$, respectively (Table 4), varied between geographical locations. The highest $\mathrm{Hd}$ was found in South Africa $(0.886 \pm 0.014)$ and Israel $(0.874 \pm 0.026)$, and the lowest in India $(0.263 \pm 0.049)$. Additionally, the sequences obtained from the worms from Israel had the highest $\pi$-value $(0.0074 \pm 0.0006)$, which was 28 times larger than the lowest $\pi$-value found in India $(0.0026 \pm$ 0.0012). In the case of S. vulpis, the $\mathrm{Hd}$ and $\pi$ were highly similar between the collection sites and ranged between $0.249-0.445$ and $0.0015-0.0024$, respectively.

Overall, 62 and 13 ITS1 haplotypes were obtained from the S. lupi and S. vulpis nematodes, respectively (Table 4). In S. lupi, 93\% (54/57) of these haplotypes were unique to a specific geographical origin, as depicted in the TCS network (Fig. 2a). However, three haplotypes were shared between worms from different geographical populations: one haplotype was found in Israeli and South African specimens; one haplotype in Israeli, South African, Indian and Australian worms; and one haplotype in Hungarian and Italian specimens. In S. vulpis, only one haplotype was shared between the nematodes from Spain, Italy and Bosnia and Herzegovina. The ML and BI trees of the ITS1 inferred two distant genetic clades, each containing only S. lupi or S. vulpis haplotypes, with high support values (bootstrap and posterior probability values of 93 and 0.997, respectively; Fig. 2b). The $S$. lupi haplotypes were sub-divided into two major genetic clades, one including Israeli, South African, Indian and Australian haplotypes, and another one including Hungarian and Italian haplotypes, with high support values (bootstrap and posterior probability values of 90 and 0.991 , respectively; Fig. 2b). ITS1 haplotype sequences were deposited in the GenBank database under the accession numbers MH630178-MH630235 for S. lupi and MH630236-MH630246 for S. vulpis.

Despite the large ITS1 intra and inter-individual variability detected, a clear differentiation between the $S$. lupi and S. vulpis specimens was evident by the PCoA plot generated with Nei's genetic distance, which mirrored the clustering obtained from the $18 S$ analysis (i.e. two S. lupi genotypes and one S. vulpis genotype; Additional file 4: Figure S3a). Moreover, when this analysis was performed including only the $S$. lupi sequences, a subdivision of genotype 1 specimens was obtained, resulting in three main clusters (Additional file 4: Figure S3b), as follows: (i) sequences from Israeli and South African specimens (genotype 1A); (ii) sequences from Indian and Australian specimens (genotype 1B); and (iii) sequences from Italian and Hungarian specimens (genotype 2). The percentage of variation explained by the axes corresponded to $88.3 \%$, separating both genotypes 1 from genotype 2, and 6.6\%, separating genotypes $1 \mathrm{~A}$ and $1 \mathrm{~B}$.

The pairwise nucleotide distances revealed a low variation within each S. lupi genotype and S. vulpis, and a high variation when comparing between these groups. When the ITS1 sequences were compared within each 


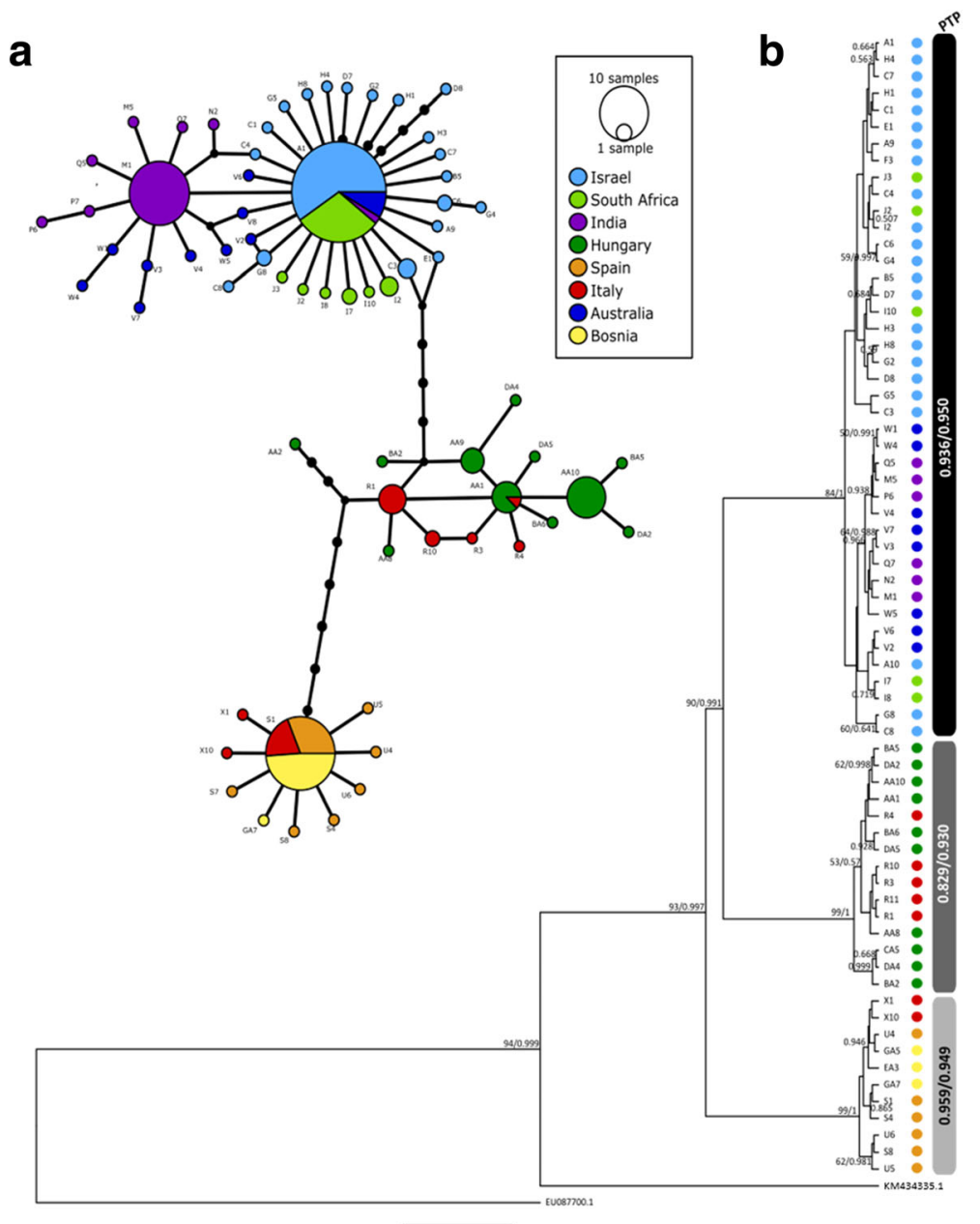

Fig. 2 Analysis of the ITS1 loci of Spirocerca spp. a Templeton-Crandall-Sing (TCS) haplotype network of the ITS1 sequences of Spirocerca spp. Each colored circle represents a haplotype; circle size is proportional to the number of sequences sharing the same haplotype. The black circles denote hypothetical sequences connecting each haplotype. b Bayesian inference tree for the ITS1 haplotypes of Spirocerca spp. Bootstrap (BS) and posterior probability (PP) values, respectively, are shown above branches. Only BS and PP values above $50 \%$ or 0.5 , respectively, are shown. Cylicospirura petrowi (KM434335.1) and Dirofilaria immitis (EU087700.1) sequences were used as the outgroups. The results of the Poisson tree processes (PTP) analysis are summarized to the right of the taxa names as grey scale bars with the support partition values found by the maximum likelihood and Bayesian solutions. The identity of each taxon in the tree and network is color-coded according to its geographical location

group (i.e. intra-genotypic variation), the median nucleotide distances were $0.73 \%$ for $S$. lupi genotypes $1 \mathrm{~A}$, ranged between $0.10-0.73 \%$ for $S$. lupi genotypes $1 \mathrm{~B}$, $0.55-1.10 \%$ for S. lupi genotype 2, and $0.37-0.74 \%$ for $S$. vulpis. The median $S$. lupi inter-genotypic variation ranged between $1.10-9.33 \%$. Genotypes $1 \mathrm{~A}$ and $1 \mathrm{~B}$ were the closest with low inter-genotypic variation (median = 1.10, 3rd IQR: $1.08-1.12$ ), and the more distantly related were genotypes $1 \mathrm{~B}$ and 2 (median $=8.06$, 3rd IQR: 7.98-8.12). Furthermore, the nucleotide distance between the S. lupi genotypes and S. vulpis ranged between 6.58-9.93\%.

Finally, the Poisson tree processes (PTP) analysis determined three putative species in the complete
Spirocerca spp. database according to the Maximum Likelihood and Bayesian solutions (observed in Fig. 2b as grayscale bars): (i) S. lupi genotype 1 (support $>0.936$ ); (ii) S. lupi genotype 2 (support $>0.829$ ); and (iii) S. vulpis (support $>0.949$ ). This analysis did not support a division within genotype 1 of $S$. lupi.

\section{Analysis of the cox 1 gene}

Two different PCRs were performed to obtain a larger sequence of the cox 1 gene (Additional file 1: Figure S1). Twenty-six of the tested 31 specimens gave positive results using both reactions. However, the $\operatorname{cox} 1$ gene from one $S$. lupi worm from Hungary, one from Israel and 
none of the worms from Australia and Italy, could be amplified by both reactions.

The analysis of the S. lupi cox 1 sequences from fragment A resulted in a clustering of the specimens with the same pattern of organization as in the $18 S$ and ITS1 analyses. Accordingly, Israeli, South African and Indian S. lupi worms grouped together (i.e. genotype 1), and apart from the Hungarian nematodes (i.e. genotype 2) and $S$. vulpis. Overall, 16 cox 1 haplotypes were obtained from the S. lupi specimens and five from S. vulpis specimens. All Israeli and South African sequences had a length of approximately $1000 \mathrm{bp}, 400 \mathrm{bp}$ larger than the expected amplicon [9] (Additional file 2: Figure S2a). The larger PCR product was a result of a $3 \mathrm{bp}$ nucleotide substitution in the targeted NTF primer sequence and the presence of an alternative $300 \mathrm{bp}$ upstream NTF region. The latter was confirmed by an alternative PCR assay using primers NTF and NTInt (Additional file 1: Figure S1, Additional file 2: Figure S2b). The pairwise nucleotide distances within $S$. lupi genotypes 1 and 2 and $S$. vulpis were lower $(2.18 \pm 1.04 \%, 0.18 \%$ and $1.06 \pm$ $0.46 \%$, respectively) than when comparing between these groups (from 5.31 to $9.34 \%$ ) (Additional file 5: Table S2). The $\operatorname{cox} 1$ sequences from the $S$. lupi specimens of genotype 1 were 97 to $99 \%$ identical to the cox 1 sequence of S. lupi complete mitochondrial genome deposited in GenBank from China (KC305876.1), while S. lupi genotype 2 sequences were $94 \%$ identical to this reference sequence. Particularly, this $S$. lupi reference sequence was more similar to the haplotypes obtained from Indian specimens (distance of $0.37-0.55 \%$ ) than to the $S$. lupi genotype 2 sequences (distance of 6.23-6.42\%). In addition, the distances between all our Spirocerca spp. to other Cylicospirura spp. ranged between 9.52-12.27\%.

The cox 1 sequences from amplified fragment B were classified in eleven haplotypes from the S. lupi specimens and three from $S$. vulpis specimens. These haplotypes grouped virtually with the same organization as obtained with the cox 1 fragment A. A higher nucleotide distance was obtained when $S$. lupi genotype 1 sequences were compared to genotype $2(7.25 \pm 0.73 \%)$ and S. vulpis (7.39 $\pm 0.87 \%)$ (Additional file 6: Table S3). Furthermore, the nucleotide distance between $S$. lupi genotype 2 and S. vulpis was $7.76 \pm 0.39$ (7.23-8.38\%). The $S$. lupi haplotypes showed a high similarity to the S. lupi reference sequences from South Africa [2] and Peru [11] (Additional file 6: Table S3). The S. lupi haplotypes of genotype 1 were $3.05 \pm 1.76$ and $3.02 \pm 1.50 \%$ different to the sequences of South African worms obtained from dogs and black-backed jackals (GenBank: KY495493.1-KY495505.1) [2] and from the Andean fox in Peru (GenBank: KY634868.1-KY634870.1) [11], respectively. Moreover, the sequences from the South African worms obtained in this study (with the exception of specimen L) were highly similar (average pairwise nucleotide distance: $0.67 \pm 0.28 \%$ ) to the sequences previously characterized from this country by Rothmann \& de Waal [2]. The South African specimen L was more closely related to $S$. lupi from Israel than to the other South African specimens. In addition, the $S$. lupi worms from Israel showed the highest similarity to the Peruvian S. lupi sequences (average pairwise nucleotide distance: $1.40 \pm 0.53 \%$ ) (Additional file 6: Table S3).

The concatenated cox 1 sequence (fragments $\mathrm{A}$ and $\mathrm{B}$ ) replicated the grouping obtained by the single cox 1 fragments (Fig. 3a-c). Sixteen haplotypes were found in S. lupi and five in S. vulpis (Table 5). There were only two shared haplotypes in the S. lupi specimens, one between three Israeli specimens and another between two Hungarian worms. The Hd was 1.00 (i.e. all sequences were different) in the nematodes obtained from S. lupi from South Africa and India and S. vulpis from Spain. Moreover, since all cox 1 sequences of worms from Bosnia and Herzegovina were identical, only one haplotype was found, and the diversity parameters were equal to 0 . Additionally, the highest values of $\pi$ were obtained from the nematodes collected from South Africa $(0.0175 \pm 0.0075)$ and Spain $(0.0149 \pm 0.0043)$ (Table 5). As obtained for the separate cox 1 fragments, the nucleotide pairwise distances were higher when comparing between the haplotypes of $S$. lupi genotypes and S. vulpis (5.91-8.63\%) than the distance within each group (0.123.55\%) (Additional file 7: Table S4, Fig. 3a). The BI and ML trees (Fig. 3b) separated with high confidence both $S$. lupi genotypes (posterior probability of 0.973 and bootstrap support of 84). Two subgroups were obtained inside the $S$. lupi genotype 1: (i) Israeli sequences and one specimen from South Africa; and (ii) the remaining sequences from South Africa and India. The S. lupi genotypes 1 and 2 were 9699\% and 93\% identical, to the mitochondrial genome of $S$. lupi from China (GenBank: KC305876.1), respectively. In addition, the $S$. lupi reference sequence from China was located next to the Indian sequences. These same observations were replicated in the haplotype network (Fig. 3c). However, the latter analysis showed an additional separation between Israeli, South African (except for one sequence which was more related to Israeli sequences) and Indian haplotypes. The newly generated sequences were deposited in the GenBank database under the accession numbers MH633995-MH634013 (Spirocerca lupi), and MH633991-MH633994 and MH634014-MH634016 (S. vulpis).

\section{SEM of specimens from Hungary}

The four specimens (two females and two males) collected from Hungary (i.e. S. lupi genotype 2) exhibited four cephalic papillae and a pair of amphids in the anterior extremity by SEM analysis. The buccal capsule did not show any teeth-like or other sclerotized structures 
a

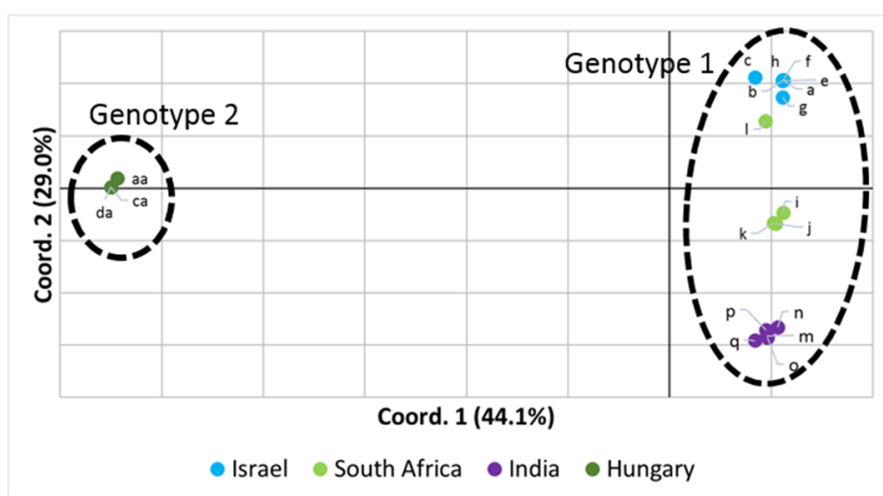

b

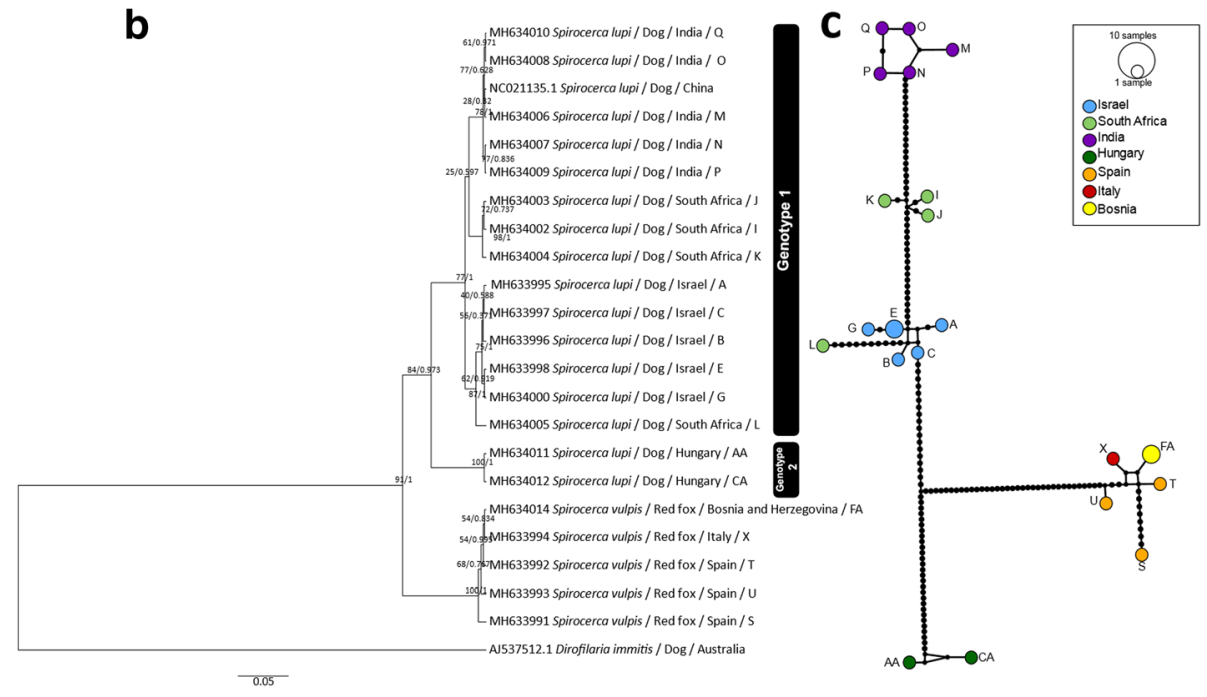

Fig. 3 Analysis of the cox1 combined fragment of Spirocerca spp. a A principal coordinate analysis (PCoA) scatter plot showing Nei's genetic distances of the cox 1 combined fragment sequences of S. lupi. b Bayesian inference tree of the cox 1 combined fragment (317 to 1201 bp) of Spirocerca spp. Bootstrap and posterior probability values are shown above branches. c Haplotype network of the cox 1 sequences. Each circle is colored according to the geographical origin of the haplotype; circle size is proportional to the number of sequences sharing the same haplotype. The black circles represent the number of mutations between the haplotypes

Table 5 Genetic diversity of the cox1 of Spirocerca spp. obtained from different geographical locations

\begin{tabular}{|c|c|c|c|c|}
\hline Geographical location & $\mathrm{S}$ & $h$ & $\mathrm{Hd} \pm \mathrm{SD}$ & $\pi \pm S D$ \\
\hline \multicolumn{5}{|l|}{ S. lupi } \\
\hline Israel $(n=7)$ & 9 & 5 & $0.857 \pm 0.137$ & $0.0042 \pm 0.0009$ \\
\hline South Africa $(n=4)$ & 19 & 4 & $1.000 \pm 0.177$ & $0.0177 \pm 0.0067$ \\
\hline India $(n=5)$ & 4 & 5 & $1.000 \pm 0.126$ & $0.0026 \pm 0.0004$ \\
\hline Hungary $(n=3)$ & 1 & 2 & $0.667 \pm 0.099$ & $0.0024 \pm 0.0011$ \\
\hline \multicolumn{5}{|l|}{ S. vulpis } \\
\hline Spain $(n=3)$ & 14 & 3 & $1.000 \pm 0.272$ & $0.0110 \pm 0.0034$ \\
\hline Bosnia and Herzegovina $(n=3)$ & 0 & 1 & 0 & 0 \\
\hline
\end{tabular}

Abbreviations: $\mathrm{n}$, number of obtained sequences; $\mathrm{S}$, segregating sites; $h$, number of haplotypes; $\mathrm{Hd}$, haplotype diversity; $\pi$, nucleotide diversity; SD, standard deviation 

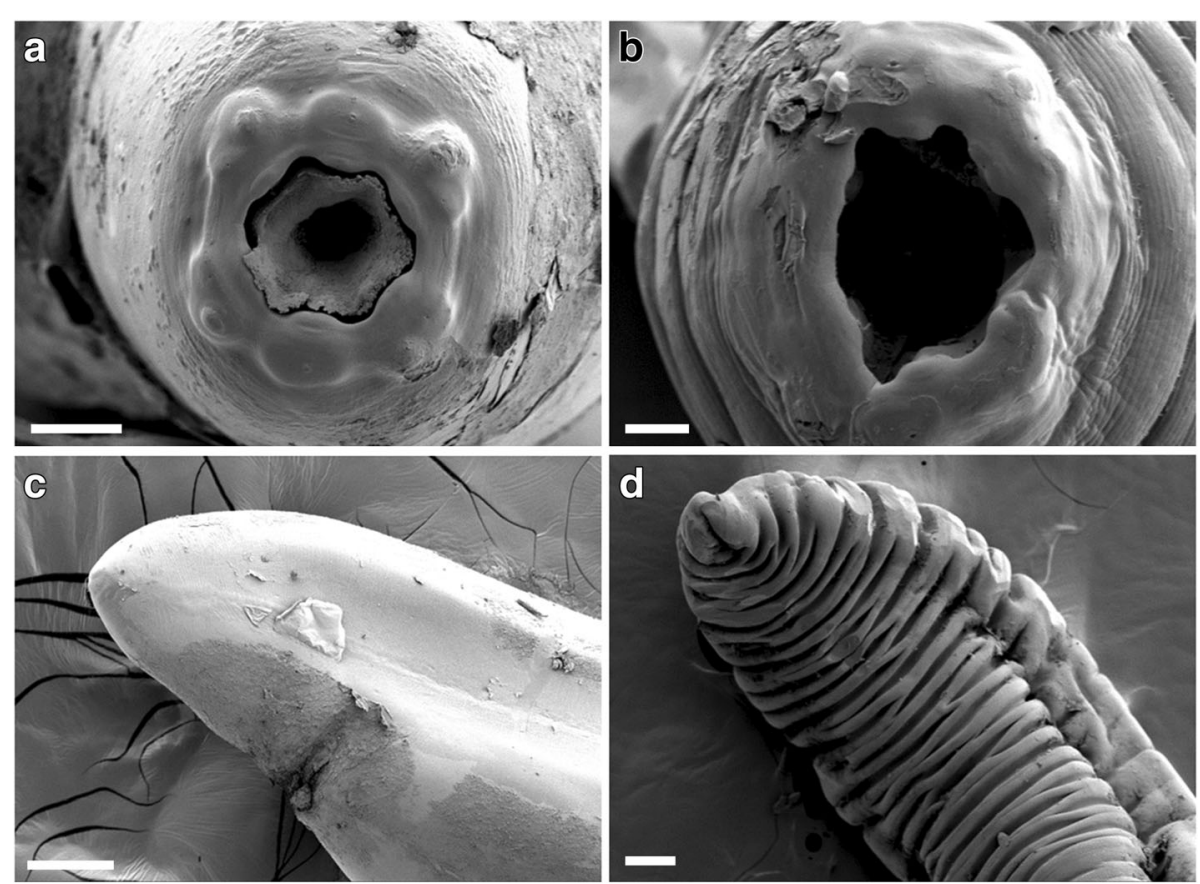

Fig. 4 Scanning electron microscopy analysis on S. lupi specimens collected in Hungary. a, b Anterior part of the worm showing four cephalic papillae, one pair of amphids and a hexagonal oral opening. $\mathbf{c}$ Anal opening observed in the posterior extremity of a female. $\mathbf{d}$ Posterior extremity of a juvenile male. Scale-bars: $\mathbf{a}, 50 \mu \mathrm{m} ; \mathbf{b}, 20 \mu \mathrm{m} ; \mathbf{c}, \mathbf{d}, 100 \mu \mathrm{m}$

(Fig. 4a, b), the main morphological characteristic that allows the differentiation between S. lupi (absent) and $S$. vulpis (present) specimens [5]. Additionally, the posterior extremity of females showed morphological similarities to both S. lupi and S. vulpis specimens (Fig. 4c). Eggs could be distinguished in different cross-sections of the worms (Additional file 8: Figure S4). The posterior extremity of both males was ventrally curved and resembled juvenile stages since neither the cloaca, spiculae, pre-anal nor post-anal papillae were evident (Fig. 4d). Furthermore, the parallel longitudinal ridges, characteristic of Spirocerca spp. males, were not observed. Instead, broad bands of approximately $30 \mu \mathrm{m}$ apart with cuticular striations separated approximately by $3 \mu \mathrm{m}$ were distinguished.

\section{Discussion}

The present study explored the phylogenetic relationships and genetic variation among S. lupi and S. vulpis collected from eight different geographical locations of four continents. With the use of ribosomal (18S, ITS1) and mitochondrial (cox1) genetic targets, different S. lupi genotypes were classified and associated with geographical distributions. Moreover, this study revealed a high intra-individual variation of the ITS1 locus in all specimens from both Spirocerca spp.

The $S$. lupi specimens were clearly separated into different genotypes according to the sampling location: genotype 1 found in specimens from Israel, South Africa,
India and Australia; and genotype 2 represented by specimens from Hungary and Italy. These findings were supported by the analysis of the highly diverse ITS1 and cox 1 loci, and the more conserved $18 S$ gene. Within $S$. lupi specimens, the ITS1 variation ranged between 0.32-8.54\%, when comparing specimens from genotype 1 with genotype 2 . A lower intra-specific variation (i.e. $0.3-2.5 \%)$ has been described in ITS1 fragments from Thelazia spp. [18], and Trichuris spp. (0.2-0.7\%) [43]. The high variation presented herein supports the presence of two clearly different genotypes, since the nucleotide distances decrease from $8.54 \%$ to $2.50 \%$ when analyzing these percentages separately according to the genotype. Moreover, the specimens from genotype 2 were classified as putative species by PTP analyses (support: 0.829 and 0.930 ). Thus, genotype 2 might be considered an intermediate entity between $S$. lupi genotype 1 and S. vulpis, as demonstrated by pairwise nucleotide distances, phylogenetic trees, haplotype networks, and the sharing of microsatellites with $S$. lupi genotype 1 and S. vulpis. The genetic distance between both S. lupi genotypes can be explained by small breeding population sizes, low infection prevalence within regions, or due to the high specificity of $S$. lupi to its definitive or intermediate hosts in each geographical location [14]. The prevalence of infection in a geographical area affects the genetic diversity of the parasite, since parasites in low prevalence regions will hardly find other genotypes 
reducing opportunities for sexual recombination. A previous study on S. lupi showed that even in geographical areas separated by $100 \mathrm{~km}$, there was lack of panmixia, probably as a result of clumped transmission of infra-populations [12]. The latter will lead to a genetic differentiation of populations from geographically separated locations after a long period of time [44], which may eventually result in allopatric speciation [14]. The large genetic difference between the S. lupi genotypes, suggested that the specimens collected from Hungarian and Italian dogs (i.e. genotype 2) could be a separate (i.e. specimens with genetic and morphological differences) or cryptic species (i.e. specimens with genetic differences but morphologically identical) [45]. However, the SEM analysis did not show evidence of morphological differences between genotype 2 specimens and $S$. lupi from Israel (i.e. genotype 1) [5], suggesting that this genotype could represent a cryptic species. Nevertheless, the morphological analysis of a larger number of sexually mature specimens collected from dogs from Hungary, Italy or Bosnia and Herzegovina or other locations in Europe is required to confirm this taxonomical status.

The ITS1 loci of Spirocerca spp. were characterized for the first time and a high genetic intra-individual variation was detected. We found that each nematode had different copies of the ITS1, with up to six different haplotypes observed per worm specimen. Intra-individual variation in the ITS1 has also been reported in Trichuris spp. [43], Echinococcus granulosus [20] and Paragonimus westermani [21]. The ITS regions are important for maintaining the secondary structure of rRNA genes and for the processing of transcribed rDNA [46]. Highly repeated sequences, such as rDNA, are maintained homogeneously within individuals and populations by concerted evolution, which assumes the continual turnover of repeats by recombination mechanisms [47]. However, sequence variation may exceed the homogenization process due to interbreeding of siblings or reduced genetic recombination [21]. Interestingly, despite the high variation found within each nematode, the ITS1 phylogenetic analyses clearly separated both $S$. lupi genotypes and differentiated them from S. vulpis. Furthermore, the variation of the ITS1 showed an additional division of the $S$. lupi genotype 1 into two groups, one containing Israeli and South African specimens and the other containing Indian and Australian ones.

South African and Israeli specimens were classified together as genotype 1A according to the ITS1 database. This finding suggests potential panmixia among populations of these two locations and highlights the possible migration of $S$. lupi-infected hosts, either definitive, intermediate or paratenic, from Africa to the Middle East or vice versa. Migration is an important evolutionary mechanism that allows subpopulations to share genetic information and contributes to adaptation to local environments [44, 48]. Indeed, the first cases of a spirocercosis outbreak in Israel were detected in the late 1980's and early 1990's in Ramat Gan, central Israel, in parasitized dogs that frequented the surroundings of a safari park that housed animals brought directly from Africa [49]. This may have been the source of this outbreak and may also explain the ITS1 genetic similarity in S. lupi populations between Israel and Africa. However, only one South African haplotype was placed next to the Israeli sequences in the cox 1 analysis, which might be explained by the different inheritance mechanisms of cox 1 and ITS1 and the genetic turnover of these loci [45]. Surprisingly, S. lupi Israeli cox 1 sequences were highly similar to a set of $S$. lupi obtained from the Andean fox (Lycalopex culpaeus) in Peru [11]. A high genetic similarity among $S$. lupi collected from two different canid hosts has been previously observed [2], with worms obtained from dogs and black-backed jackals in South Africa sharing two haplotypes, and thus suggesting the transmission between those canid species that live in sympatry. Moreover, our analysis shows that high similarity between specimens can be evident even when collected from two canids that are not in the same geographical area. This situation might also reflect the migration of infected hosts between both geographical locations [44].

The cox 1 locus showed a high genetic interspecific variation and diversity, as demonstrated by the nucleotide differences between $S$. lupi genotypes and S. vulpis $(0.17-11.07 \%)$ and the haplotype diversity within each species. For this gene, almost every nematode specimen contributed with one sequence variant, and only three worms from Israel and two from Hungary shared the same variant. Previous studies have addressed the variability in the cox 1 gene of $S$. lupi. For instance, Traversa et al. [9] reported two haplotypes in 20 adult worms collected from undetermined hosts, one haplotype from specimens from Israel, Iran and South Africa, and another from Austrian and Italian specimens, with only six nucleotide substitutions separating them [9]; these findings correlate to genotypes 1 and 2 found in this study. However, we found a greater variation, with 19 haplotypes in total and 89 subtitutions within all S. lupi specimens, of which 30 contributed to the separation of the two genotypes. In addition, there were subgroups within each genotype according to geographical areas, since low $\operatorname{cox} 1$ pairwise nucleotide distances were found among the worms from the same country (0.263$0.827 \%$ ), and higher distances were detected when comparing between locations (1.418-3.546\%). Furthermore, a previous study of $S$. lupi conducted in 60 worms collected from 20 dogs confined to $300 \mathrm{~km}^{2}$ in South Africa found 11 haplotypes and a low genetic differentiation using the $\operatorname{cox} 1$ locus [10], which supports our results of 
low diversity within geographical locations. Finally, Rothmann \& de Waal [2] reported 11 haplotypes in 49 nematodes from 31 dogs from South Africa. The difference in the number of haplotypes found in the latter study and ours might rely on the algorithms used for haplotype phasing, of which some are more strict than others [50]. Another possibility may be due to the sampling of sibling worms which tend to inbreed, reducing the genetic variability [14]. However, in our case, even sibling worms had different haplotypes, which highlights the large genetic variability within the studied nematodes.

Studies on the genetic diversity and differentiation between parasites are of special importance since they can expand the understanding of their distribution patterns and processes of speciation [14]. There is still limited information regarding the evolutionary history and speciation processes in S. lupi and S. vulpis, contrary to knowledge on other helminth parasites, such as Fasciola hepatica [8], Fascioloides magna [51] and Anisakis spp. [52]. For instance, a high genetic diversity within each host but low differentiation between populations was found in F. hepatica, with the implications of possible spread of anthelmintic resistance genes. Additionally, a migratory route of hosts infected with $F$. magna and a speciation foci was found in a genetic population study [51]. Spirocerca spp. have been suggested as parasites of domestic dogs that have spilled over to wildlife [53]. The latter assumption has been based on reports of $S$. lupi-like life stages found in other canid species [54-58]. However, most of these reports lack detailed morphological and genotypic screening, thus, it cannot be ruled out that the observed nematodes may represent different S. lupi genotypes or different Spirocerca spp. For instance, morphometric and phylogenetic analyses have led to the description of $S$. vulpis collected from red foxes, which had been previously misclassified as S. lupi, based on macroscopic and microscopic observations of the worms [59]. Therefore, many questions regarding the speciation and evolutionary history of Spirocerca spp. rise with our results, including if this genus originated in fact from dogs and spread to wildlife animals, or the spread of this infection occurred in the opposite direction.

\section{Conclusions}

A high genetic variability of Spirocerca spp. was found in specimens from Asia, Africa, Europe, and Oceania based on the analysis of the ITS1 and cox1 loci. This included a high degree of variation in the ITS1 loci with up to six haplotypes in a single worm and marked polymorphism in the cox 1 gene of nematodes from different origins. The high genetic diversity found herein led to the identification of two S. lupi genotypes which have different geographical distributions. This study suggests that migration and low prevalence in each geographical location studied may have been the driving forces of diversification with the genus Spirocerca.

\section{Additional files}

Additional file 1: Figure S1. Diagram of primers employed for the amplification of different fragments of the 185 (a), ITS1 (b) and cox1 (c) loci. PCR with primers NTF and NTInt was used for the confirmation of a 300 bp upstream NTF binding regions in South African and Israeli specimens. All amplicons obtained in the PCRs were sequenced. (TIF $397 \mathrm{~kb}$ )

Additional file 2: Figure S2.Agarose gels stained with ethidium bromide showing the $\sim 600$ and 1000 bp amplicons obtained during the amplification of the cox 1 fragment A using the NTF and NTR primers (a) and the $\sim 300 \mathrm{bp}$ amplicons after running PCR using the NTF and NTInt primers (b). (TIF $1837 \mathrm{~kb}$ )

Additional file 3: Table S1. Pairwise nucleotide distance of the 185 gene (1611 bp fragment) expressed as percentages between specimens of Spirocerca spp. and other nematodes of the order Spirurida. (DOCX 18 $\mathrm{kb})$

Additional file 4: Figure S3. Principal coordinate analysis ( $P C O A)$ scatter plot showing Nei's genetic distances of the ITS1 sequences of S. Iupi (a) and Spirocerca spp. (b) according to the sampling locations. The percentage of total variation attributed to each axis is indicated next to each coordinate. Each specimen is represented as a color-coded circle according to the geographical origin. (TIF $385 \mathrm{~kb}$ )

Additional file 5: Table S2. Pairwise nucleotide differences (\%) between cox1 fragment A (317 to 967 bp) haplotypes obtained from S. lupi and S. vulpis, and reference cox1 sequences of Spirocerca spp. and Cylicospirura spp. available on GenBank. (DOCX 22 kb)

Additional file 6: Table S3.Pairwise nucleotide differences (\%) between cox1 fragment B (807 to 1201 bp) haplotypes obtained from S. lupi and S. vulpis, and cox 1 reference sequences of S. lupi and D. immitis available on GenBank. (DOCX 31 kb)

Additional file 7: Table S4.Pairwise nucleotide distance (\%) in the cox1 (317 to $1201 \mathrm{bp}$ ) haplotypes of Spirocerca spp. (DOCX 16 kb)

Additional file 8: Figure S4. Scanning electron microscopy of a S. lupi female from Hungary showing the eggs in a uterus cross-section. (TIF 2174 kb)

\section{Abbreviations}

AIC: Akaike information criterion; BI: Bayesian inference; bPTP: Bayesian solution for the Poisson tree processes; cox1: Cytochrome $c$ oxidase subunit 1; ESS: Effective sample size; $h$ : Number of haplotypes; Hd: Haplotype diversity; HKY: Hasegawa-Kishino-Yano; ITS1: Internal transcribed spacer 1; MCMC: Markov chain Monte Carlo; ML: Maximum-likelihood;

MPTP: Maximum likelihood for the Poisson Tree Processes; PCR: Polymerasechain reaction; PTP: Poisson Tree Processes; rDNA: Ribosomal DNA;

S: Segregating sites; SEM: Scanning electron microscopy; TCS: TempletonCrandall-Sing haplotype network; TIM: Transitional model; TPM: Three parameter model; UPW: Ultrapure water; 18S: Small ribosomal subunit; $\pi$ : Nucleotide diversity

\section{Acknowledgements}

The authors thank Ricardo Gutierrez for fruitful discussions. Ian Beveridge, Amer Alic, Adnan Hodzic, Carlos Martínez-Carrasco and Gloria SanchisMonsonis for providing the specimens from Australia, Bosnia and Herzegovina and Spain.

\section{Funding}

The study was funded by GB's lab internal funds and a partial stipend granted to AR by the University of Costa Rica.

\section{Availability of data and materials}

The data supporting the conclusions of this article are provided within the article and its additional files. The original datasets are available upon 
request to the corresponding author. The generated sequences of S. lupi were deposited in the GenBank database under the accession numbers MH628159 (18S), MH630178-MH630235 (ITS1) and MH633995-MH634013 (cox1). The generated sequences of S. vulpis were deposited in the GenBank database under the accession numbers MH630236-MH630246 (ITS1), and MH633991-MH633994 and MH634014-MH634016 (cox1).

\section{Authors' contributions}

$A R$ and $G B$ conceived and designed the study. AR performed all DNA extraction, PCR and phylogenetic analyses. ED, RF, AJ, SB, KS, AM and DO collected worm specimens. $A R$ and $G B$ analyzed and interpreted the results. $A R, E D, R F, A J, S B, K S, A M, D O$ and $G B$ wrote the draft of the manuscript. All authors read and approved the final manuscript.

\section{Ethics approval and consent to participate}

Not applicable.

\section{Consent for publication}

Not applicable.

\section{Competing interests}

The authors declare that they have no competing interests.

\section{Publisher's Note}

Springer Nature remains neutral with regard to jurisdictional claims in published maps and institutional affiliations.

\section{Author details}

${ }^{1}$ Koret School of Veterinary Medicine, The Hebrew University of Jerusalem, Rehovot, Israel. ${ }^{2}$ Department of Animal Sciences, Tel-Hai Academic College, Upper Galilee, Israel. ${ }^{3}$ Department of Parasitology and Zoology, University of Veterinary Medicine, Budapest, Hungary. ${ }^{4}$ Department of Veterinary Medicine, College of Veterinary Sciences and Animal Husbandry, Central Agricultural University, Mizoram, India. ${ }^{5}$ Department of Veterinary Parasitology, College of Veterinary Sciences and Animal Husbandry, Central Agricultural University, Mizoram, India. ${ }^{6}$ Department of Veterinary Biosciences, Melbourne Veterinary School, The University of Melbourne, Victoria, Australia. ${ }^{7}$ Kimron Veterinary Institute, Bet Dagan, Israel. ${ }^{8}$ Department of Veterinary Medicine, University of Bari, Valenzano, Italy.

\section{Received: 9 August 2018 Accepted: 19 November 2018}

\section{Published online: 14 December 2018}

\section{References}

1. van der Merwe LL, Kirberger RM, Clift S, Williams M, Keller N, Naidoo V. Spirocerca lupi infection in the dog: a review. Vet J. 2008;176:294-309.

2. Rothmann W, de Waal PJ. Diversity of Spirocerca lupi in domestic dogs and black-backed jackals (Canis mesomelas) from South Africa. Vet Parasitol. 2017;244:59-63

3. Pence DB, Windberg LA. Population dynamics across selected habitat variables of the helminth community in coyotes, Canis latrans, from south Texas. J Parasitol. 1984;70:735-46.

4. Murray M. Incidence and pathology of Spirocerca lupi in Kenya. J Comp Pathol. 1968;78:401-5

5. Rojas A, Sanchis-Monsonis G, Alic A, Hodzic A, Otranto D, Yasur-Landau D, et al. Spirocerca vulpis sp. nov. (Spiruridae: Spirocercidae): description of a new nematode species of the red fox, Vulpes vulpes (Carnivora: Canidae). Parasitology. 2018;21:1-12.

6. Makouloutou P, Rana HB, Adhikari B, Devkota B, Dhakal IP, Sato H. A distinct genetic population of Gongylonema pulchrum from water buffaloes in Nepal. J Parasitol. 2013;99:669-76.

7. Hauquier F, Leliaert F, Rigaux A, Derycke S, Vanreusel A. Distinct genetic differentiation and species diversification within two marine nematodes with different habitat preference in Antarctic sediments. BMC Evol Biol. 2017;17:120.

8. Beesley NJ, Williams DJ, Paterson S, Hodgkinson J. Fasciola hepatica demonstrates high levels of genetic diversity, a lack of population structure and high gene flow: possible implications for drug resistance. Int J Parasitol. 2017:47:11-20

9. Traversa D, Costanzo F, lorio R, Aroch I, Lavy E. Mitochondrial cytochrome c oxidase subunit 1 (cox1) gene sequence of Spirocerca lupi (Nematoda, Spirurida): avenues for potential implications. Vet Parasitol. 2007;146:263-70.
10. de Waal PJ, Gous A, Clift SJ, Greeff JM. High within-host genetic variation of the nematode Spirocerca lupi in a high-density urban dog population. Vet Parasitol. 2012;187:259-66.

11. Gomez-Puerta L, Carrasco J, Lopez-Urbina M, Gonzalez A. Morphological and molecular identification of Spirocerca lupi (Nematoda: Spiruridae) found in the Andean fox (Lycalopex culpaeus). J Par Dis. 2018:42:449-54.

12. Greeff JM, Reid K, Gagjee JR, Clift S, de Waal P. Population genetic structure of the parasitic nematode Spirocerca lupi in South Africa. Vet Parasitol. 2018;258:64-9.

13. Al-Sabi MN, Hansen MS, Chriél M, Holm E, Larsen G, Enemark HL. Genetically distinct isolates of Spirocerca sp. from a naturally infected red fox (Vulpes vulpes) from Denmark. Vet Parasitol. 2014;205:389-96.

14. Huyse T, Poulin R, Théron A. Speciation in parasites: a population genetics approach. Trends Parasitol. 2005:21:469-75.

15. Nadler SA, Pérez-Ponce de León G. Integrating molecular and morphological approaches for characterizing parasite cryptic species: implications for parasitology. Parasitology. 2011;138:1688-709.

16. Bik HM, Fournier D, Sung W, Bergeron RD, Thomas WK. Intra-genomic variation in the ribosomal repeats of nematodes. PLoS One. 2013;8:e78230.

17. Eickbush TH, Eickbush DG. Finely orchestrated movements: evolution of the ribosomal RNA genes. Genetics. 2007;175:477-85.

18. Otranto D, Traversa D. Molecular characterization of the first internal transcribed spacer of ribosomal DNA of the most common species of eyeworms (Thelazioidea: Thelazia). J Parasitol. 2004;90:185-8.

19. Conole JC, Chilton NB, Järvis T, Gasser RB. Intraspecific and interspecific variation in the second internal transcribed spacer (ITS-2) sequence for Metastrongylus (Nematoda: Metastrongyloidea) detected by high resolution PCR-RFLP. Int J Parasitol. 1999;29:1935-40.

20. Bowles J, Blair D, McManus DP. A molecular phylogeny of the genus Echinococcus. Parasitology. 1995;110:317-28.

21. van Herwerden L, Blair D, Agatsuma T. Intra- and interindividual variation in ITS1 of Paragonimus westermani (Trematoda: Digenea) and related species: implications for phylogenetic studies. Mol Phylogenet Evol. 1999;12:67-73.

22. Makouloutou P, Setsuda A, Yokoyama M, Tsuji T, Saita E, Torii H, et al. Genetic variation of Gongylonema pulchrum from wild animals and cattle in Japan based on ribosomal RNA and mitochondrial cytochrome c oxidase subunit I genes. J Helminthol. 2013:87:326-35.

23. Floyd RM, Rogers AD, Lambshead PJD, Smith CR. Nematode-specific PCR primers for the 18S small subunit rRNA gene. Mol Ecol Notes. 2005;5:611-2.

24. Vrain $T$, Wakarchuk $D$, Lévesque $A$, Hamilton $R$. Intraspecific rDNA restriction fragment lenght polymorphism in the Xiphinema americanum group. Fund Appl Nematol. 1992;15:563-73.

25. Cherry T, Szalanski AL, Todd TC, Powers TO. The internal transcribed spacer region of Belonolaimus (Nemata: Belonolaimidae). J Nematol. 1997;29:23-9.

26. Boutsika K, Brown DJF, Phillips MS, Blok VC. Molecular characterization of the ribosomal DNA of Paratrichodorus macrostylus, P. pachydermus, Trichodorus primitivus and T. similis (Nematoda: Trichodoridae). Nematology. 2004;6:641-54

27. Liu GH, Wang Y, Song HQ, Li MW, Ai L, Yu XL, et al. Characterization of the complete mitochondrial genome of Spirocerca lupi: sequence, gene organization and phylogenetic implications. Parasit Vectors. 2013;6:45.

28. Casiraghi M, Anderson TJ, Bandi C, Bazzocchi C, Genchi C. A phylogenetic analysis of filarial nematodes: comparison with the phylogeny of Wolbachia endosymbionts. Parasitology. 2001;122:93-103.

29. Ye J, Coulouris G, Zaretskaya I, Cutcutache I, Rozen S, Madden TL. Primer-BLAST: a tool to design target-specific primers for polymerase chain reaction. BMC Bioinformatics. 2012;13:134.

30. Bowles J, Blair D, DP MM. Genetic variants within the genus Echinococcus identified by mitochondrial DNA sequencing. Mol Biochem Parasitol. 1992;54:165-73.

31. Kumar S, Stecher G, Tamura K. MEGA7: Molecular Evolutionary Genetics Analysis Version 7.0 for bigger datasets. Mol Biol Evol. 2016;33:1870-4.

32. Otranto D, Tarsitano E, Traversa D, Giangaspero A, De Luca F, Puccini V. Differentiation among three species of bovine Thelazia (Nematoda: Thelaziidae) by polymerase chain reaction-restriction fragment length polymorphism of the first internal transcribed spacer ITS-1 (rDNA). Int J Parasitol. 2001;31:1693-8.

33. Librado P, Rozas J. DnaSP v5: a software for comprehensive analysis of DNA polymorphism data. Bioinformatics. 2009:25:1451-2.

34. Nei M. Genetic distance between populations. Am Nat. 1972;106:283-92.

35. Peakall R, Smouse PE. GenAlEx 6.5: genetic analysis in Excel. Population genetic software for teaching and research - an update. Bioinformatics. 2012;28:2537-9. 
36. Clement M, Posada D, Crandall KA. TCS: a computer program to estimate gene genealogies. Mol Ecol. 2000;9:1657-9.

37. Darriba D, Taboada GL, Doallo R, Posada D. jModelTest 2: more models, new heuristics and parallel computing. Nat Methods. 2012;9:772.

38. Miller MA, Pfreiffer W, Schwartz T. Creating the CIPRES Science Gateway for inference of large phylogenetic trees. In: Gateway Computing Environments Workshop (GCE); 2010. p. 1-8.

39. Guindon S, Dufayard JF, Lefort V, Anisimova M, Hordijk W, Gascuel O. New algorithms and methods to estimate maximum-likelihood phylogenies: assessing the performance of PhyML 3.0. Syst Biol. 2010;59:307-21.

40. Lefort V, Longueville JE, Gascuel O. SMS: Smart Model Selection in PhyML. Mol Biol Evol. 2017;34:2422-4.

41. Zhang J, Kapli P, Pavlidis P, Stamatakis A. A general species delimitation method with applications to phylogenetic placements. Bioinformatics. 2013:29:2869-76

42. Rojas A, Segev G, Markovics A, Aroch I, Baneth G. Detection and quantification of Spirocerca lupi by HRM qPCR in fecal samples from dogs with spirocercosis. Parasit Vectors. 2017;10:435

43. Callejon R, Halajian A, de Rojas M, Marrugal A, Guevara D, Cutillas C. 165 partial gene mitochondrial DNA and internal transcribed spacers ribosomal DNA as differential markers of Trichuris discolor populations. Vet Parasitol. 2012;186:350-63.

44. Barrett LG, Thrall PH, Burdon JJ, Linde CC. Life history determines genetic structure and evolutionary potential of host-parasite interactions. Trends Ecol Evol. 2008;23:678-85.

45. Pérez-Ponce de León G, Nadler SA. What we don't recognize can hurt us: a plea for awareness about cryptic species. J Parasitol. 2010;96:453-64.

46. van Nues RW, Rientjes JM, Morré SA, Mollee E, Planta RJ, Venema J, et al. Evolutionarily conserved structural elements are critical for processing of Internal Transcribed Spacer 2 from Saccharomyces cerevisiae precursor ribosomal RNA. J Mol Biol. 1995;250:24-36.

47. Ganley AR, Kobayashi T. Highly efficient concerted evolution in the ribosomal DNA repeats: total rDNA repeat variation revealed by wholegenome shotgun sequence data. Genome Res. 2007;17:184-91.

48. Yamamichi $\mathrm{M}$, Innan $\mathrm{H}$. Estimating the migration rate from genetic variation data. Heredity (Edinb). 2012;108:362-3.

49. Mazaki-Tovi M, Baneth G, Aroch I, Harrus S, Kass PH, Ben-Ari T, et al. Canine spirocercosis: clinical, diagnostic, pathologic, and epidemiologic characteristics. Vet Parasitol. 2002;107:235-50.

50. Browning SR, Browning BL. Haplotype phasing: existing methods and new developments. Nat Rev Genet. 2011;12:703-14.

51. Juhasova L, Kralova-Hromadova I, Bazsalovicsova E, Minarik G, Stefka J, Mikulicek P, et al. Population structure and dispersal routes of an invasive parasite, Fascioloides magna, in North America and Europe. Parasit Vectors. 2016;9:547.

52. Mattiucci S, Cipriani P, Levsen A, Paoletti M, Nascetti G. Molecular epidemiology of Anisakis and anisakiasis: an ecological and evolutionary road map. Adv Parasitol. 2018;99:93-263.

53. Weinstein SB, Lafferty KD. How do humans affect wildlife nematodes? Trends Parasitol. 2015;31:222-7.

54. Alexander AB, Poirotte C, Porton IJ, Freeman KL, Rasambainarivo F, Olson KG, et al. Gastrointestinal parasites of captive and free-living lemurs and domestic carnivores in eastern Madagascar. J Zoo Wildl Med. 2016;47:141-9.

55. Szafrańska E, Wasielewski O, Bereszyński A. A faecal analysis of helminth infections in wild and captive wolves, Canis lupus L., in Poland. J Helminthol. 2010;84:415-9.

56. Meshgi B, Eslami A, Bahonar A, Kharrazian-Moghadam M, Gerami-Sadeghian A. Prevalence of parasitic infections in the red fox (Vulpes vulpes) and golden jackal (Canis aureus) in Iran. Iran J Vet Res. 2009;10:387-91.

57. Blume GR, Reis Junior JL, Gardiner CH, Hoberg EP, Pilitt PA, Monteiro RV, et al. Spirocerca lupi granulomatous pneumonia in two free-ranging maned wolves (Chrysocyon brachyurus) from central Brazil. J Vet Diagn Invest. 2014;26:815-7.

58. Rinas MA, Nesnek R, Kinsella JM, DeMatteo KE. Fatal aortic aneurysm and rupture in a neotropical bush dog (Speothos venaticus) caused by Spirocerca lupi. Vet Parasitol. 2009:164:347-9.
59. Segovia J, Miquel J, Feliu C, Torres J. Morphological and morphometric study of Spirocerca lupi. Helminthologia. 2001;38:115-21.

60. Ferguson JA, Woodberry K, Gillin C, Jackson DH, Sanders JL, Madigan W, et al. Cylicospirura species (Nematoda: Spirocercidae) and stomach nodules in cougars (Puma concolor) and bobcats (Lynx rufus) in Oregon. J Wildlife Dis. 2011:47:140-53.

\section{Ready to submit your research? Choose BMC and benefit from:}

- fast, convenient online submission

- thorough peer review by experienced researchers in your field

- rapid publication on acceptance

- support for research data, including large and complex data types

- gold Open Access which fosters wider collaboration and increased citations

- maximum visibility for your research: over $100 \mathrm{M}$ website views per year

At BMC, research is always in progress.

Learn more biomedcentral.com/submissions 